\title{
Functional polymorphisms in microRNAs and susceptibility to liver cancer: a meta-analysis and meta-regression
}

\author{
B.S. Wang, Z. Liu, W.X. Xu and S.L. Sun \\ Department of General Surgery, \\ Shengjing Hospital of China Medical University, Shenyang, China \\ Corresponding author: B.S. Wang \\ E-mail: cmu2h_wbs@126.com
}

Genet. Mol. Res. 13 (3): 5426-5440 (2014)

Received June 5, 2013

Accepted October 1, 2013

Published July 24, 2014

DOI http://dx.doi.org/10.4238/2014.July.24.22

\begin{abstract}
MicroRNAs (miRNAs) are small non-coding RNA molecules that play a fundamental role in controlling a variety of biological functions. Emerging evidence has shown that common genetic polymorphisms in miRNAs may be associated with the development of liver cancer; however, several individually published studies showed inconclusive results. This meta-analysis aimed to derive a more precise estimation of the association between functional polymorphisms in miRNAs and susceptibility to liver cancer. A literature search of PubMed, Embase, Web of Science, and China BioMedicine (CBM) databases was conducted on articles published before May 1, 2012. Crude odds ratios with $95 \%$ confidence intervals were calculated. Fourteen case-control studies were included with a total of 6824 liver cancer patients and 7674 healthy controls. Nine single nucleotide polymorphisms in miRNAs were assessed, including miR-146a $\mathrm{G}>\mathrm{C}$ (rs2910164), miR-499 T>C (rs3746444), miR-218 A > G (rs11134527), miR-let-7c Ins/Del (rs6147150), miR-106b-25 A>G (rs999885), miR34b/c T >C (rs4938723), miR-196a-2 C > T (rs11614913), miR-920 Ins/ Del (rs16405), and miR-122 Ins/Del (rs3783553). The meta-analysis
\end{abstract}


results showed that miR-let-7c*Del, miR-34b/c*C, and miR-122*Del variants may be associated with increased liver cancer risk. Conversely, miR-920*Del variant may decrease the risk of liver cancer. However, miR-146a G>C, miR-196a-2 C $>$ T, miR-499 T>C, and miR-218 A > G polymorphisms showed no significant association with liver cancer risk. In conclusion, the current meta-analysis suggests that miR-let$7 \mathrm{c} *$ Del, miR-34b/c*C and miR-122*Del variants may be associated with increased liver cancer risk, while miR-920*Del variant may be a protective factor against liver cancer.

Key words: Liver cancer; Single nucleotide polymorphism; MicroRNA; Meta-analysis

\section{INTRODUCTION}

Liver cancer is the sixth most frequently diagnosed cancer and the third leading cause of cancer death worldwide, with an estimated 748,000 new liver cancer cases and 696,000 deaths during 2008 (Thun et al., 2010; Jemal et al., 2011). The major risk factors for liver cancer include chronic hepatitis B virus (HBV) and hepatitis C virus infections, dietary aflatoxin exposure, alcohol-related cirrhosis, fatty liver disease, obesity, smoking, diabetes, and iron overload (Liu et al., 2012a). Although chronic HBV infection has been recognized as the most important causal factor for liver cancer in humans, only a small fraction of HBV-persistent carriers eventually develop liver cancer, suggesting that genetic variations may play roles in the carcinogenesis of liver cancer after HBV infection (Wang et al., 2012b). Therefore, the role of common genetic variations is increasingly recognized as an important factor in determining an individual's susceptibility to liver cancer (El-Serag and Rudolph, 2007).

MicroRNAs (miRNAs) are small non-coding RNA molecules with approximately 23 nucleotides, which act as post-transcriptional regulators of gene expression and have been implicated in the initiation and progression of various cancers (Kim et al., 2012; Wang et al., 2012a). Therefore, it has been hypothesized that genetic polymorphisms in miRNAs can be functional and may be associated with the development of liver cancer (Ryan et al., 2010). Numerous case-control studies have demonstrated that single nucleotide polymorphisms (SNPs) in miRNAs, including rs2910164 $(\mathrm{G}>\mathrm{C})$ in miR-146a, rs $11134527(\mathrm{~A}>\mathrm{G})$ in miR-218, rs11614913 (C>T) in miR-196a-2, rs999885 (A>G) in miR-106b-25, and rs3783553 (Ins/Del) in miR-122, may contribute to increased susceptibility to liver cancer (Xu et al., 2008; Gao et al., 2009; Qi et al., 2010; Liu et al., 2012b; Zhang et al., 2012). However, some other studies have suggested that functional polymorphisms in miRNAs are not associated with susceptibility to liver cancer (Akkiz et al., 2011bc; Zhang et al., 2011; Zhou et al., 2012). A recent metaanalysis of 9 case-control studies by Wang et al. (2012c) revealed that two common SNPs rs2910164 in miR-146a and rs11614913 in miR-196a-2 were not associated with the risk of liver cancer. Similar results were obtained from the other two previous meta-analyses, which focused on the effects of rs2910164 and rs11614913 polymorphisms on cancer susceptibility (Qiu et al., 2011; Xu et al., 2011a). Conversely, a meta-analysis based on 15 independent casecontrol studies conducted by Guo et al. (2012) suggested that rs11614913 in miR-196a-2 may contribute to increased liver cancer risk. In view of these conflicting results from previous 
studies and the insufficient statistical power in the previous meta-analyses, we performed the present meta-analysis to update previous meta-analyses, as well to provide a more comprehensive and reliable conclusion on the association between functional polymorphisms in miRNAs and susceptibility to liver cancer.

\section{MATERIAL AND METHODS}

\section{Literature search}

Relevant studies published before May 1, 2013 were identified through a search in PubMed, Embase, Web of Science, and China BioMedicine (CBM) databases using the following terms: ("genetic polymorphism" or "polymorphism" or "SNP" or "single nucleotide polymorphism" or "gene mutation" or "genetic variants") and ("liver neoplasms" or "liver cell adenoma" or "hepatocellular carcinoma" or "hepatic neoplasms" or "liver cancer" or "hepatic cancer" or "liver tumor" or "hepatic tumor" or "hepatic carcinogenesis") and ("microRNAs" or "microRNA" or "miRNAs" or "miRNA" or "primary microRNA" or "primary miRNA" or "pri-miRNA" or "small temporal RNA" or "pre-miRNA"). The references from the eligible articles or textbooks were also reviewed to find other potential sources. Disagreements were resolved through discussions between the authors.

\section{Inclusion and exclusion criteria}

Studies included in our meta-analysis had to meet the following criteria: a) case-control studies focused on the association between functional polymorphisms in miRNAs and liver cancer risk; b) all patients diagnosed with liver cancer were confirmed by pathological or histological examinations; c) published data about the frequencies of alleles or genotypes were sufficient. Studies were excluded when they were: a) not a case-control study on the association between functional polymorphisms in miRNAs and liver cancer risk; b) duplicates of previous publications; c) publications with incomplete data; d) meta-analyses, letters, reviews, or editorial articles. If more than one study by the same author using the same case series was published, either the study with the largest sample size or the most recently published study was included.

\section{Data extraction}

Data from the published studies were extracted independently by two authors and entered on a standardized form. For each study, the following characteristics and numbers were collected: the first author, year of publication, country, language, study design, ethnicity of subjects, numbers of subjects, gender ratio, mean age, detecting sample, genotype method, allele and genotype frequencies of SNP, and evidence of Hardy-Weinberg equilibrium (HWE). In cases of conflicting evaluations, disagreements were resolved through discussions between the authors.

\section{Quality assessment of studies included}

Two authors independently assessed the quality of studies included according to the 
modified STROBE-quality score systems (Gallo et al., 2012). Forty assessment items related to quality appraisal were used in this meta-analysis with scores ranging from 0 to 40 . The studies included were classified into three levels based on their scores: low quality (0-19), moderate quality (20-29), and high quality (30-40). Disagreements were also resolved through discussions between the authors.

\section{Statistical analysis}

Crude odds ratios (ORs) with $95 \%$ confidence intervals $(95 \% \mathrm{CI})$ were calculated under five genetic models: allele, dominant, recessive, homozygous, and heterozygous models. The statistical significance of the pooled ORs was examined using the Z-test. Between-study variations and heterogeneities were estimated using the Cochran Q-statistic with $\mathrm{P}<0.05$ as a cutoff to identify statistically significant heterogeneity (Higgins and Thompson, 2002). We also quantified the effects of heterogeneity by using the $I^{2}$ test (ranging from 0 to $100 \%$ ), which represents the proportion of inter-study variability that can be contributed to heterogeneity rather than to chance (Zintzaras and Ioannidis, 2005). A significant Q-test with $\mathrm{P}<0.05$ or $I^{2}>$ $50 \%$ indicated that heterogeneity between studies existed, and then, the random-effect model (DerSimonian Laird method) was conducted for the meta-analysis; otherwise, the fixed-effect model (Mantel-Haenszel method) was used. To explore the potential sources of heterogeneity, multivariate meta-regression analysis was used (Ioannidis et al., 2008). Subgroup analysis was performed by the ethnicity and genotype method. Sensitivity analysis was performed by omitting each study in turn to assess the quality and consistency of the results. Begger funnel plots were used to detect publication bias. The Egger linear regression test was also used to evaluate publication bias (Peters et al., 2006). We tested whether genotype frequencies were in HWE using the $\chi^{2}$ test. All the P values were two-sided. All analyses were calculated using the STATA Version 12.0 software (Stata Corp., College Station, TX, USA).

\section{RESULTS}

\section{Characteristics of the studies included}

Overall, 148 potentially relevant studies were identified by searching electronic databases. According to the inclusion criteria, 14 case-control studies (Gao et al., 2009; Chen et al., 2010; Qi et al., 2010; Akkiz et al., 2011a,b,c; Xu et al., 2008, 2011b; Liu et al., 2012b; Xiang et al., 2012; Yu et al., 2012; Zhang et al., 2011, 2012; Zhou et al., 2012) were included in this meta-analysis and 134 were excluded. The flow chart of the study selection process is shown in Figure 1. A total of 14,516 subjects were involved in this meta-analysis, including 6824 liver cancer patients and 7674 healthy controls. The publication years of the studies considered ranged from 2008 to 2012. All patients diagnosed with liver cancer were confirmed by pathological examinations. The sources of controls in all studies included were based on the general population (population-based). The DNA samples used for examination of genetic polymorphisms in miRNAs were extracted from blood in all studies included. Genotyping methods included direct DNA sequencing, TaqMan, primer-introduced restriction analysispolymerase chain reaction (PIRA-PCR), PCR-ligase detection reaction (PCR-LDR) and PCRrestriction fragment length polymorphism (PCR-RFLP). The HWE test was conducted on the 
genotype distribution of the controls in all studies included. None of the studies deviated from the HWE (all P > 0.05). All quality scores of studies included were higher than 20 (moderatehigh quality). The characteristics and methodological quality of the studies included are summarized in Table 1.

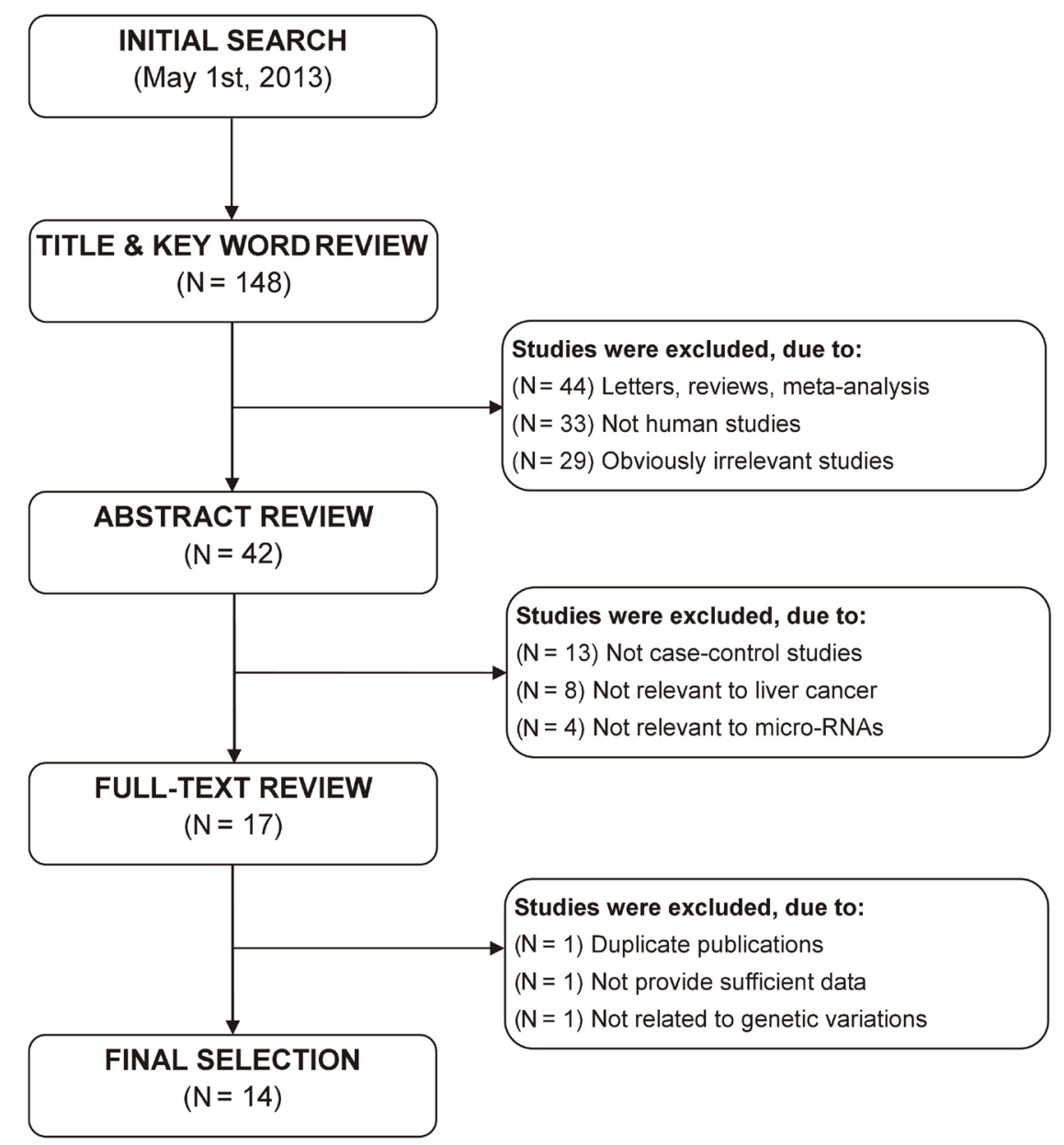

Figure 1. Flow chart of literature search and study selection. Fourteen case-control studies were included in this meta-analysis.

\section{Quantitative data synthesis}

Nine SNPs in miRNAs were addressed, including miR-146a G>C (rs2910164), miR$499 \mathrm{~T}>\mathrm{C}$ (rs3746444), miR-218 A $>$ G (rs11134527), miR-let-7c Ins/Del (rs6147150), miR106b-25 A > G (rs999885), miR-34b/c T $>$ C (rs4938723), miR-196a-2 C $>$ T (rs11614913), miR920 Ins/Del (rs16405), and miR-122 Ins/Del (rs3783553). A summary of the meta-analysis findings of the association between genetic polymorphisms in miRNAs and liver cancer risk is provided in Table 2 . 


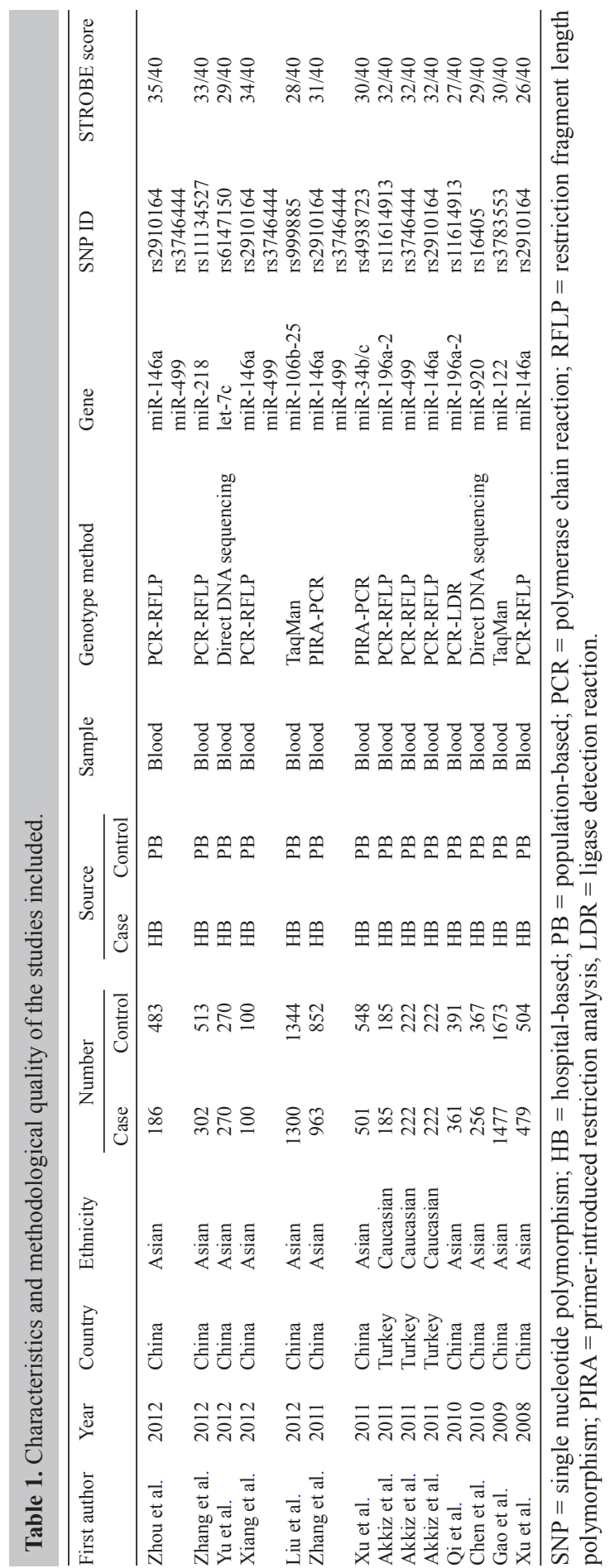


B.S. Wang et al.

5432

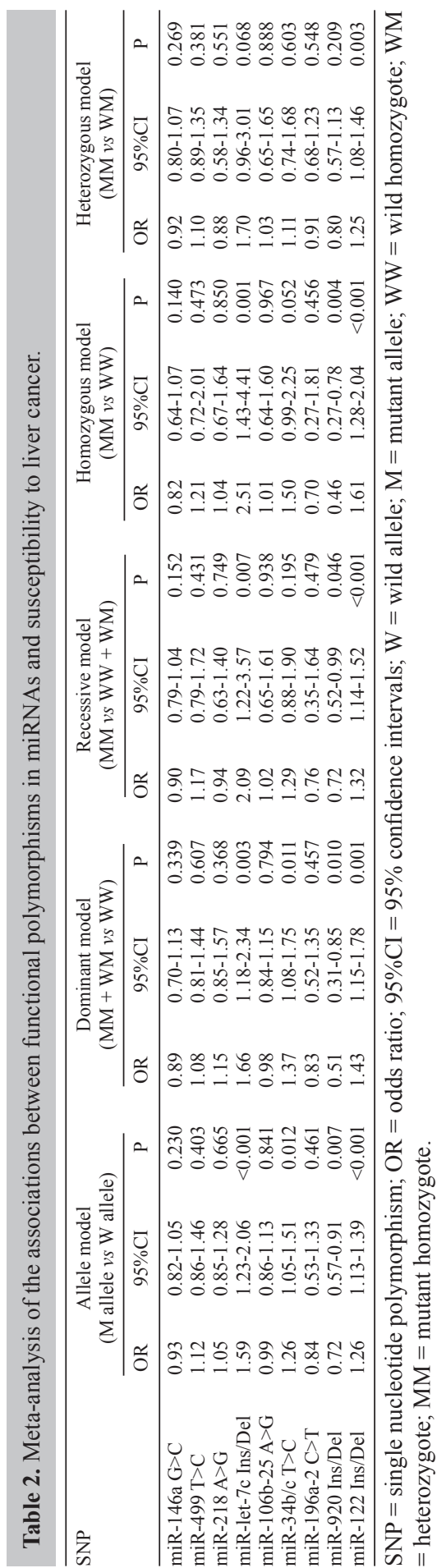

Genetics and Molecular Research 13 (3): 5426-5440 (2014) 
The meta-analysis results showed that miR-let-7c*Del variant was associated with increased liver cancer risk (Del allele $v s$ Ins allele: $\mathrm{OR}=1.59,95 \% \mathrm{CI}=1.23-2.06, \mathrm{P}<0.001$; $\mathrm{Del} / \mathrm{Del}+\mathrm{Ins} / \mathrm{Del} v s \mathrm{Ins} / \mathrm{Ins}: \mathrm{OR}=1.66,95 \% \mathrm{CI}=1.18-2.34, \mathrm{P}=0.003 ; \mathrm{Del} / \mathrm{Del} v s \mathrm{Ins} / \mathrm{Ins}+$ Ins/Del: OR $=2.09,95 \% \mathrm{CI}=1.22-3.57, \mathrm{P}=0.007 ; \mathrm{Del} / \mathrm{Del} v s \mathrm{Ins} / \mathrm{Ins}: \mathrm{OR}=2.51,95 \% \mathrm{CI}=$ 1.43-4.41, $\mathrm{P}=0.001$ ) (Figure 2). Furthermore, $\mathrm{miR}-34 \mathrm{~b} / \mathrm{c} * \mathrm{C}$ variant also showed significant association with increased liver cancer risk ( $\mathrm{C}$ allele $v s \mathrm{~T}$ allele: $\mathrm{OR}=1.26,95 \% \mathrm{CI}=1.05$ $1.51, \mathrm{P}=0.012 ; \mathrm{CC}+\mathrm{TC} v s \mathrm{TT}: \mathrm{OR}=1.37,95 \% \mathrm{CI}=1.08-1.75, \mathrm{P}=0.011)$. We also found that miR-122*Del variant could be a risk factor for liver cancer susceptibility (Del allele vs Ins allele: $\mathrm{OR}=1.26,95 \% \mathrm{CI}=1.13-1.39, \mathrm{P}<0.001 ; \mathrm{Del} / \mathrm{Del}+\mathrm{Ins} / \mathrm{Del} v s \mathrm{Ins} / \mathrm{Ins}: \mathrm{OR}=1.43$, $95 \% \mathrm{CI}=1.15-1.78, \mathrm{P}=0.001 ; \mathrm{Del} / \mathrm{Del} v s \mathrm{Ins} / \mathrm{Ins}+\mathrm{Ins} / \mathrm{Del}: \mathrm{OR}=1.32,95 \% \mathrm{CI}=1.14-1.52, \mathrm{P}$ $<0.001 ; \mathrm{Del} / \mathrm{Del}$ vs Ins/Ins: OR = 1.61, 95\%CI $=1.28-2.04, \mathrm{P}<0.001 ; \mathrm{Del} / \mathrm{Del} v s$ Ins/Del: OR $=1.25,95 \% \mathrm{CI}=1.08-1.46, \mathrm{P}=0.003)$. Conversely, miR-920*Del variant was associated with decreased liver cancer risk (Del allele $v s$ Ins allele: $\mathrm{OR}=0.72,95 \% \mathrm{CI}=0.57-0.91, \mathrm{P}=0.007$; $\mathrm{Del} / \mathrm{Del}+\mathrm{Ins} / \mathrm{Del} v s \mathrm{Ins} / \mathrm{Ins}: \mathrm{OR}=0.51,95 \% \mathrm{CI}=0.31-0.85, \mathrm{P}=0.010 ; \mathrm{Del} / \mathrm{Del} v s \mathrm{Ins} / \mathrm{Ins}+$ Ins/Del: OR $=0.72,95 \% \mathrm{CI}=0.52-0.99, \mathrm{P}=0.046 ; \mathrm{Del} / \mathrm{Del} v s$ Ins/Ins: $\mathrm{OR}=0.46,95 \% \mathrm{CI}=$ 0.27-0.78, $\mathrm{P}=0.004$ ). However, miR-146a G>C, miR-196a-2 $\mathrm{C}>\mathrm{T}$, miR-499 $\mathrm{T}>\mathrm{C}$, and miR$218 \mathrm{~A}>\mathrm{G}$ polymorphisms were not significantly associated with susceptibility to liver cancer (all $\mathrm{P}>0.05$ ) (Figure 3).

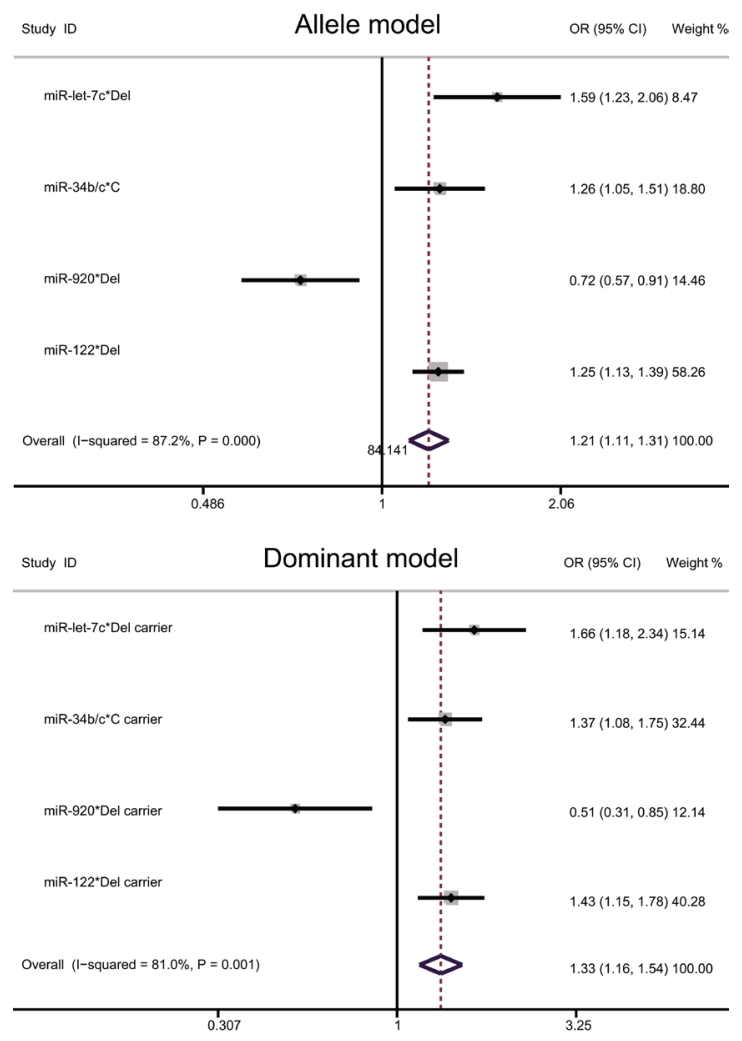

Figure 2. Forest plot of ORs for the associations of miR-let-7c Ins/Del, miR-34b/c T $>$ C, miR-122 Ins/Del, and miR-920 Ins/Del with susceptibility to liver cancer. 


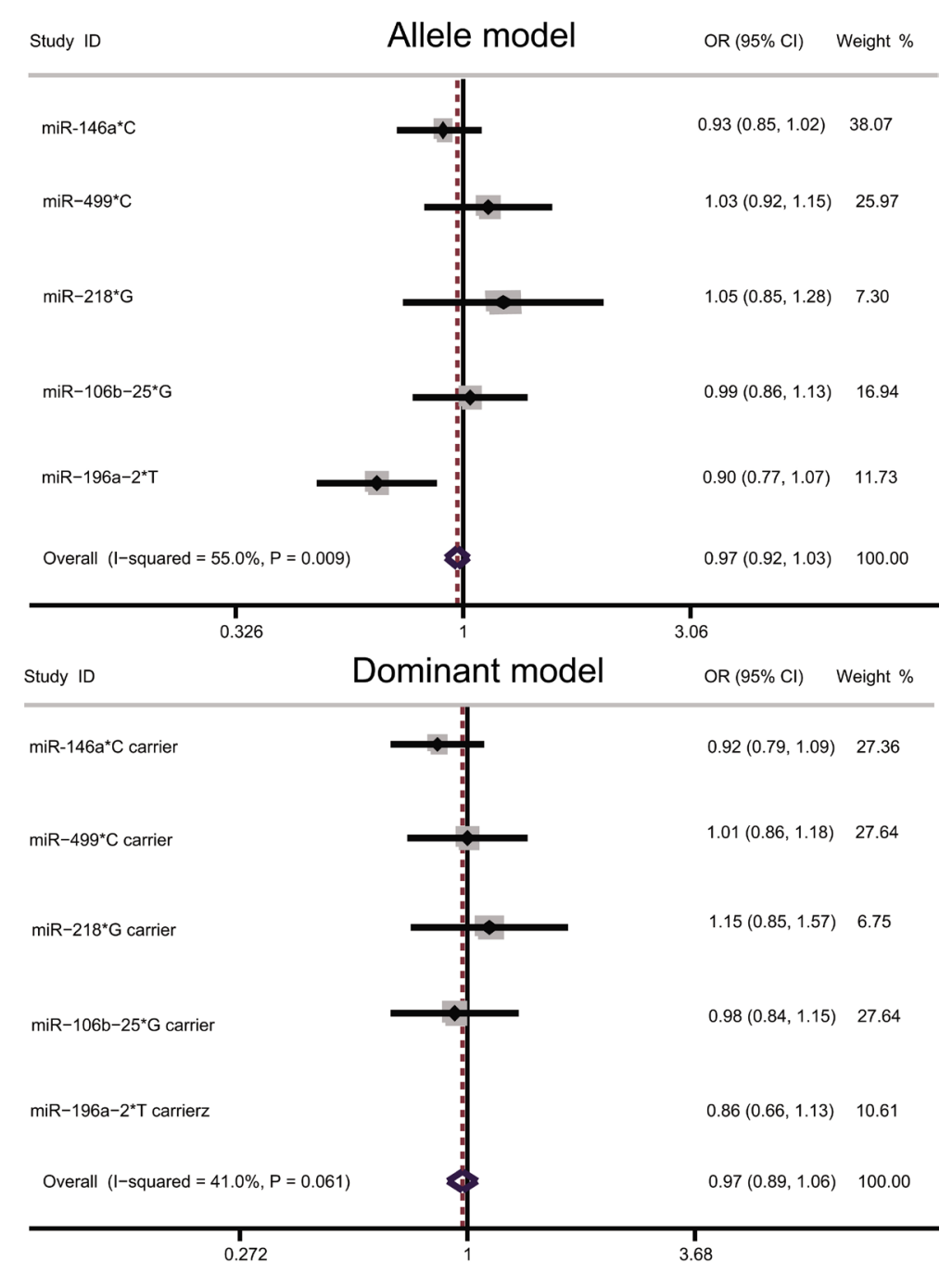

Figure 3. Forest plot of ORs for the associations of miR-146a G>C, miR-196a-2 C $>$ T, miR-499 T $>$ C, and miR-218 $A>G$ with susceptibility to liver cancer.

In searching for possible factors that might have impacted the results, further subgroup analyses for two common SNPs miR-146a G $>$ C and miR-499 $\mathrm{T}>\mathrm{C}$ were performed based on the ethnicity and genotype method. The results also showed that there was no significant association between miR-146a G $>C$ and miR-499 $\mathrm{T}>\mathrm{C}$ polymorphisms and liver cancer risk (all $\mathrm{P}>0.05$ ) (as shown in Table 3).

\section{Meta-regression and sensitivity analyses}

Multivariate meta-regression analysis was used to explore the possible sources of heterogeneity among studies for miR-146a G $>$ C and miR-499 $\mathrm{T}>\mathrm{C}$. 
Genetic polymorphisms in microRNAs and liver cancer

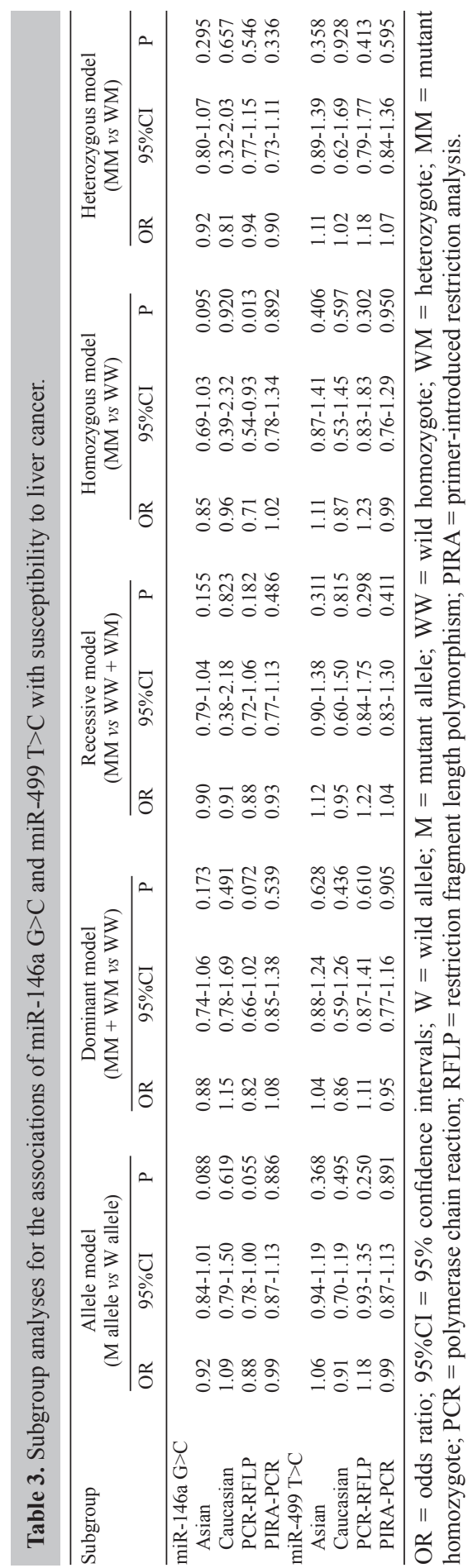


The results showed that none of these five potential heterogeneity factors explained heterogeneity (as shown in Table 4). Sensitivity analyses were performed to assess the influence of each individual study on the pooled ORs by omitting individual studies in turn. The analysis results suggested that no individual study significantly affected the pooled ORs of miR-146a $\mathrm{G}>\mathrm{C}$ and miR$499 \mathrm{~T}>\mathrm{C}$ polymorphisms under the allele model (Figure 4), indicating statistically robust results.

\begin{tabular}{|c|c|c|c|c|c|c|}
\hline \multirow[t]{2}{*}{ Heterogeneity factors } & \multirow[t]{2}{*}{ Coefficient } & \multirow[t]{2}{*}{ SE } & \multirow[t]{2}{*}{ Z } & \multirow[t]{2}{*}{$\mathrm{P}$} & \multicolumn{2}{|c|}{$95 \% \mathrm{CI}$} \\
\hline & & & & & UL & LL \\
\hline \multicolumn{7}{|l|}{ Publication year } \\
\hline miR-146a G $>C$ & -0.02 & 0.07 & -0.32 & 0.753 & -0.16 & 0.12 \\
\hline $\operatorname{miR}-499 \mathrm{~T}>\mathrm{C}$ & 0.12 & 0.11 & 1.12 & 0.262 & -0.09 & 0.334 \\
\hline \multicolumn{7}{|l|}{ Country } \\
\hline $\operatorname{miR}-146 a \mathrm{G}>\mathrm{C}$ & 0.11 & 0.07 & 1.47 & 0.142 & -0.04 & 0.25 \\
\hline $\operatorname{miR}-499 \mathrm{~T}>\mathrm{C}$ & 0.29 & 0.26 & 1.15 & 0.251 & -0.21 & 0.79 \\
\hline \multicolumn{7}{|l|}{ Ethnicity } \\
\hline $\mathrm{miR}-146 \mathrm{a} \mathrm{G}>\mathrm{C}$ & 0.04 & 0.23 & 0.18 & 0.860 & -0.42 & 0.50 \\
\hline $\operatorname{miR}-499 \mathrm{~T}>\mathrm{C}$ & -0.63 & 0.95 & -0.66 & 0.506 & -2.48 & 1.23 \\
\hline \multicolumn{7}{|l|}{ Genotype method } \\
\hline $\operatorname{miR}-146 a \mathrm{G}>\mathrm{C}$ & -0.28 & 0.23 & -1.18 & 0.237 & -0.73 & 0.18 \\
\hline $\operatorname{miR}-499 \mathrm{~T}>\mathrm{C}$ & -0.04 & 0.63 & -0.06 & 0.952 & -1.27 & 1.19 \\
\hline \multicolumn{7}{|l|}{ STROBE score } \\
\hline $\mathrm{miR}-146 \mathrm{a} \mathrm{G}>\mathrm{C}$ & -0.03 & 0.04 & -0.85 & 0.397 & -0.10 & 0.04 \\
\hline $\operatorname{miR}-499 \mathrm{~T}>\mathrm{C}$ & -0.01 & 0.07 & -0.09 & 0.931 & -0.14 & 0.13 \\
\hline
\end{tabular}

$\mathrm{SE}=$ standard error; $95 \% \mathrm{CI}=95 \%$ confidence interval; $\mathrm{UL}=$ upper limit; $\mathrm{LL}=$ lower limit.
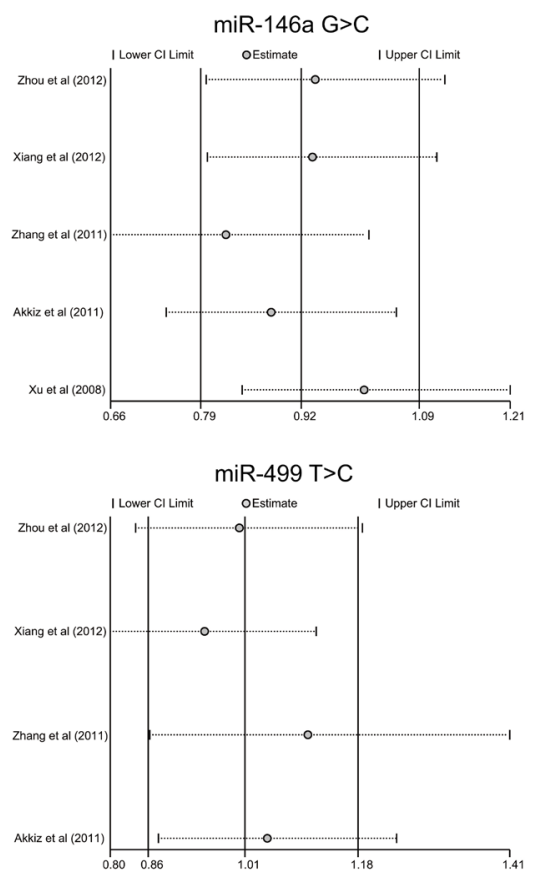

Figure 4. Sensitivity analysis of the pooled ORs on the associations of miR-146a G $>C$ and miR-499 $T>C$ with susceptibility to liver cancer under the dominant model. Results were computed by omitting each study in turn. The two ends of the dotted lines represent the $95 \% \mathrm{CI}$. 


\section{Publication bias}

Publication bias within available research results may not be representative of all research results. Begger's funnel plot and the Egger linear regression test were performed to assess publication bias in the included studies. The shapes of the funnel plots of the association of miR-146a G>C and miR-499 $\mathrm{T}>\mathrm{C}$ with liver cancer risk under the dominant model did not reveal any evidence of obvious asymmetry (Figure 5). The Egger test also did not indicate any statistically significant evidence of publication bias under the dominant model (miR-146a: $t=$ -1.12, $\mathrm{P}=0.346$; miR-499: $t=1.27, \mathrm{P}=0.331$ ).
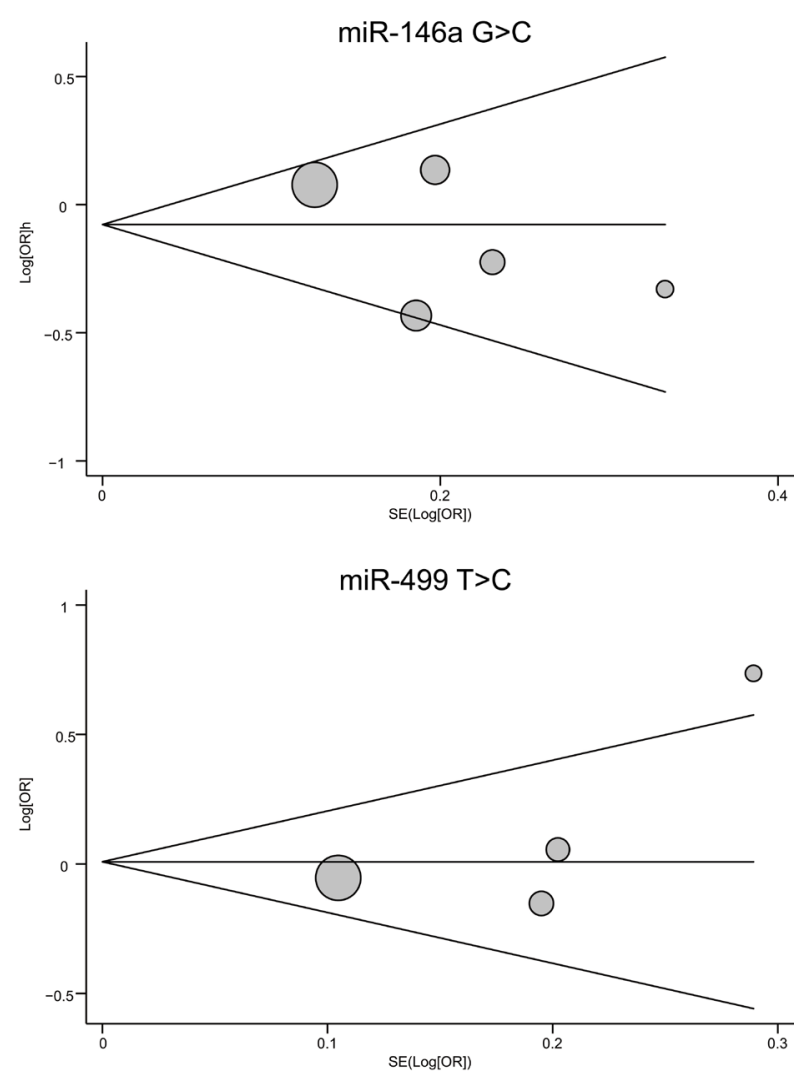

Figure 5. Begger's funnel plot of publication biases on the associations of miR-146a $G>C$ and miR-499 $T>C$ with susceptibility to liver cancer under the dominant model. Each point represents a separate study for the indicated association. $\log [\mathrm{OR}]=$ natural logarithm of OR. Horizontal line $=$ mean magnitude of the effect.

\section{DISCUSSION}

Human miRNAs, as endogenous small non-coding RNAs, play a fundamental role in controlling a variety of biological functions, such as developmental patterning, cell differentiation, cell proliferation, genome rearrangements and transcriptional regulation (He and Hannon, 2004; Kota et al., 2009). A growing body of evidence has demonstrated that miRNAs 
possess the functions essential for normal development and cellular homeostasis, and therefore, dysfunction of these molecules has been associated with several human cancers, including liver cancer (Ryan et al., 2010). Thus, genetic mutations in miRNAs may contribute to its abnormal expression and could probably be linked to liver cancer risk (Negrini et al., 2009). Several miRNA genes have been studied in the aspect of correlations with genetic susceptibility to liver cancer, involving miR-146a, miR-499, miR-218, miR-let-7c, miR-106b-25, etc. (Croce, 2009). These miRNAs have long been recognized as targets of genomic lesions, which can frequently activate oncogenes and inactivate tumor suppressors in liver cancer cells such as amplification, deletion, and epigenetic silencing through critical functions downstream of oncogene or tumor suppressor signaling pathways (Ferracin et al., 2010). Some previous casecontrol studies and meta-analyses have suggested that common genetic variants in miRNAs may play important roles in the development of liver cancer (Gao et al., 2009; Qi et al., 2010; Xu et al., 2011a; Guo et al., 2012; Liu et al., 2012b; Zhang et al., 2012), while other investigations have not found any convincing evidence of these polymorphisms in increasing susceptibility to liver cancer (Akkiz et al., 2011b,c; Qiu et al., 2011; Xu et al., 2011a; Zhang et al., 2011; Wang et al., 2012c; Zhou et al., 2012). This controversy could be explained by several reasons, such as the differences in study designs, sample size, ethnicity of subjects, source of controls, genotype methods, etc. Considering this controversy, we performed the present meta-analysis to provide a more comprehensive and reliable conclusion on the association between functional polymorphisms in miRNAs and susceptibility to liver cancer.

In this meta-analysis, 14 case-control studies were included with a total of 6824 liver cancer cases and 7674 healthy controls. When all the eligible studies were pooled into the meta-analysis, the results indicated that miR-let-7c*Del, miR-34b/c*C and miR-122*Del variants were associated with increased liver cancer risk, while miR-920*Del variant was associated with a decreased liver cancer risk. Although the exact function of miRNAs in liver cancer is not yet clear, a possible reason could be that inherited mutations in miRNAs are associated with changes in their expression and function and could thereby explain inter-individual differences in susceptibility to liver cancer (Adams et al., 2007). However, miR-146a G>C, miR-196a-2 C $>$ T, miR-499 T $>$ C, and miR-218 A $>$ G showed no significant association with liver cancer risk. These findings are partially consistent with most previous studies and metaanalyses, indicating that these polymorphisms may not be major determinants or promoting factors in the development of liver cancer in humans. In subgroup analysis based on the ethnicity and genotyping method for two common SNPs miR-146a G $>C$ and miR-499 T $>$ C, we also found no significant association between these polymorphisms and liver cancer risk.

Similar to other meta-analyses, our study also bears some limitations and drawbacks. First, there were only fourteen articles included in the present meta-analysis, so the sample size was relatively small and may not provide sufficient statistical power. Therefore, more studies with a larger sample size are still needed to provide a more representative statistical analysis. Second, as a type of retrospective study, a meta-analysis may encounter recall or selection bias, possibly influencing the reliability of our study results (Ioannidis and Lau, 1999). Third, our lack of access to the original data from the studies limited further evaluation of potential interactions between other factors and liver cancer risks, such as gene-environment and gene-gene interactions (Dennis et al., 2011).

In conclusion, our meta-analysis suggests that miR-let- $7 \mathrm{c}^{*} \mathrm{Del}, \mathrm{miR}-34 \mathrm{~b} / \mathrm{c} * \mathrm{C}$ and miR-122*Del variants may be associated with increased liver cancer risk, while miR-920*Del 
variant may be a preventive factor against liver cancer. These relationships have the potential to provide functional profiling of miRNA genes involved in the development of liver cancer. Our meta-analysis may also serve as a guide for further studies in the diagnosis and clinical therapy of liver cancer. Considering the limitations mentioned above, detailed studies are still needed to confirm our findings. Further studies investigating the effect of gene-environment interactions on liver cancer are also required.

\section{ACKNOWLEDGMENTS}

Research supported by the Science Foundation of Science and Technology Bureau of Liaoning Province of China (\#2008225008-8).

\section{REFERENCES}

Adams BD, Furneaux H and White BA (2007). The micro-ribonucleic acid (miRNA) miR-206 targets the human estrogen receptor-alpha (ERalpha) and represses ERalpha messenger RNA and protein expression in breast cancer cell lines. Mol. Endocrinol. 21: 1132-1147.

Akkiz H, Bayram S, Bekar A, Akgollu E, et al. (2011a). A functional polymorphism in pre-microRNA-196a-2 contributes to the susceptibility of hepatocellular carcinoma in a Turkish population: a case-control study. J. Viral Hepat. 18: e399-e407.

Akkiz H, Bayram S, Bekar A, Akgollu E, et al. (2011b). Genetic variation in the microRNA-499 gene and hepatocellular carcinoma risk in a Turkish population: lack of any association in a case-control study. Asian Pac. J. Cancer Prev. 12: 3107-3112.

Akkiz H, Bayram S, Bekar A, Akgollu E, et al. (2011c). No association of pre-microRNA-146a rs2910164 polymorphism and risk of hepatocellular carcinoma development in Turkish population: a case-control study. Gene 486: 104-109.

Chen S, He Y, Ding J, Jiang Y, et al. (2010). An insertion/deletion polymorphism in the 3' untranslated region of betatransducin repeat-containing protein (beta $\operatorname{TrCP}$ ) is associated with susceptibility for hepatocellular carcinoma in Chinese. Biochem. Biophys. Res. Commun. 391: 552-556.

Croce CM (2009). Causes and consequences of microRNA dysregulation in cancer. Nat. Rev. Genet. 10: 704-714.

Dennis J, Hawken S, Krewski D, Birkett N, et al. (2011). Bias in the case-only design applied to studies of geneenvironment and gene-gene interaction: a systematic review and meta-analysis. Int. J. Epidemiol. 40: 1329-1341.

El-Serag HB and Rudolph KL (2007). Hepatocellular carcinoma: epidemiology and molecular carcinogenesis. Gastroenterology 132: 2557-2576.

Ferracin M, Veronese A and Negrini M (2010). Micromarkers: miRNAs in cancer diagnosis and prognosis. Expert Rev. Mol. Diagn. 10: 297-308.

Gallo V, Egger M, McCormack V, Farmer PB, et al. (2012). Strengthening the Reporting of observational studies in Epidemiology: Molecular Epidemiology STROBE-ME. An extension of the STROBE statement. J. Epidemiol. Community Health 66: 844-854.

Gao Y, He Y, Ding J, Wu K, et al. (2009). An insertion/deletion polymorphism at miRNA-122-binding site in the interleukin-1alpha 3' untranslated region confers risk for hepatocellular carcinoma. Carcinogenesis 30: 2064-2069.

Guo J, Jin M, Zhang M and Chen K (2012). A genetic variant in miR-196a2 increased digestive system cancer risks: a meta-analysis of 15 case-control studies. PLoS One 7: e30585.

He L and Hannon GJ (2004). MicroRNAs: small RNAs with a big role in gene regulation. Nat. Rev. Genet. 5: 522-531.

Higgins JP and Thompson SG (2002). Quantifying heterogeneity in a meta-analysis. Stat. Med. 21: 1539-1558.

Ioannidis JP and Lau J (1999). Pooling research results: benefits and limitations of meta-analysis. Jt. Comm J. Qual. Improv. 25: 462-469.

Ioannidis JP, Patsopoulos NA and Rothstein HR (2008). Reasons or excuses for avoiding meta-analysis in forest plots. BMJ 336: 1413-1415.

Jemal A, Bray F, Center MM, Ferlay J, et al. (2011). Global cancer statistics. CA Cancer J. Clin. 61: 69-90.

Kim WH, Min KT, Jeon YJ, Kwon CI, et al. (2012). Association study of microRNA polymorphisms with hepatocellular carcinoma in Korean population. Gene 504: 92-97.

Kota J, Chivukula RR, O’Donnell KA, Wentzel EA, et al. (2009). Therapeutic microRNA delivery suppresses tumorigenesis in a murine liver cancer model. Cell 137: 1005-1017. 
Liu Y, Pan S, Liu L, Zhai X, et al. (2012a). A genetic variant in long non-coding RNA HULC contributes to risk of HBVrelated hepatocellular carcinoma in a Chinese population. PLoS One 7: e35145.

Liu Y, Zhang Y, Wen J, Liu L, et al. (2012b). A genetic variant in the promoter region of miR-106b-25 cluster and risk of HBV infection and hepatocellular carcinoma. PLoS One 7: e32230.

Negrini M, Nicoloso MS and Calin GA (2009). MicroRNAs and cancer - new paradigms in molecular oncology. Curr. Opin. Cell Biol. 21: 470-479.

Peters JL, Sutton AJ, Jones DR, Abrams KR, et al. (2006). Comparison of two methods to detect publication bias in metaanalysis. JAMA 295: 676-680.

Qi P, Dou TH, Geng L, Zhou FG, et al. (2010). Association of a variant in MIR 196A2 with susceptibility to hepatocellular carcinoma in male Chinese patients with chronic hepatitis B virus infection. Hum. Immunol. 71: 621-626.

Qiu LX, Wang Y, Xia ZG, Xi B, et al. (2011). miR-196a2 C allele is a low-penetrant risk factor for cancer development. Cytokine 56: 589-592.

Ryan BM, Robles AI and Harris CC (2010). Genetic variation in microRNA networks: the implications for cancer research. Nat. Rev. Cancer 10: 389-402.

Thun MJ, DeLancey JO, Center MM, Jemal A, et al. (2010). The global burden of cancer: priorities for prevention. Carcinogenesis 31: 100-110.

Wang J, Wang Q, Liu H, Shao N, et al. (2012a). The association of miR-146a rs2910164 and miR-196a2 rs11614913 polymorphisms with cancer risk: a meta-analysis of 32 studies. Mutagenesis 27: 779-788.

Wang X, Zhang X, Qiu B, Tang Y, et al. (2012b). MDM2 SNP309T $>$ G polymorphism increases susceptibility to hepatitis B virus-related hepatocellular carcinoma in a northeast Han Chinese population. Liver Int. 32: 1172-1178.

Wang Z, Cao Y, Jiang C, Yang G, et al. (2012c). Lack of association of two common polymorphisms rs2910164 and rs11614913 with susceptibility to hepatocellular carcinoma: a meta-analysis. PLoS One 7: e40039.

Xiang Y, Fan S, Cao J, Huang S, et al. (2012). Association of the microRNA-499 variants with susceptibility to hepatocellular carcinoma in a Chinese population. Mol. Biol. Rep. 39: 7019-7023.

Xu T, Zhu Y, Wei QK, Yuan Y, et al. (2008). A functional polymorphism in the miR-146a gene is associated with the risk for hepatocellular carcinoma. Carcinogenesis 29: 2126-2131.

Xu W, Xu J, Liu S, Chen B, et al. (2011a). Effects of common polymorphisms rs11614913 in miR-196a2 and rs2910164 in miR-146a on cancer susceptibility: a meta-analysis. PLoS One 6: e20471.

$\mathrm{Xu}$ Y, Liu L, Liu J, Zhang Y, et al. (2011b). A potentially functional polymorphism in the promoter region of miR-34b/c is associated with an increased risk for primary hepatocellular carcinoma. Int. J. Cancer 128: 412-417.

Yu Q, Zhou CX, Chen NS, Zheng SD, et al. (2012). A polymorphism within ErbB4 is associated with risk for hepatocellular carcinoma in Chinese population. World J. Gastroenterol. 18: 383-387.

Zhang LS, Liang WB, Gao LB, Li HY, et al. (2012). Association between pri-miR-218 polymorphism and risk of hepatocellular carcinoma in a Han Chinese population. DNA Cell Biol. 31: 761-765.

Zhang XW, Pan SD, Feng YL, Liu JB, et al. (2011). Relationship between genetic polymorphism in microRNAs precursor and genetic predisposition of hepatocellular carcinoma. Zhonghua Yu Fang Yi. Xue. Za Zhi. 45: 239-243.

Zhou J, Lv R, Song X, Li D, et al. (2012). Association between two genetic variants in miRNA and primary liver cancer risk in the Chinese population. DNA Cell Biol. 31: 524-530.

Zintzaras E and Ioannidis JP (2005). Heterogeneity testing in meta-analysis of genome searches. Genet. Epidemiol. 28: 123-137. 